\title{
Immunoglobulin G4-related disease masquerading as tonsil carcinoma
}

\author{
TL Chow ${ }^{1}$ *, FRCS (Edin), FHKAM (Surgery), Nancy WF Yuen², FRCPath, FHKAM (Pathology), Wilson WY Kwan', FRCS (Edin), \\ FHKAM (Surgery), CY Choi ${ }^{1}$, FRCS (Edin), FHKAM (Surgery)
}

Departments of ${ }^{1}$ Surgery and ${ }^{2}$ Anatomical Pathology, United Christian Hospital, Kwun Tong, Hong Kong

*Corresponding author: chowt|@ha.org.hk / tamlinc@yahoo.com

Hong Kong Med J 2017;23:537-8

DOI: 10.12809/hkmj164842

\section{Case report}

An 85-year-old man presented with a history of odynophagia since March 2015. He was a chronic smoker but did not drink alcohol. He had a medical history of hypertension, diabetes mellitus, and gout. He was first seen by us in April 2015. At the first presentation, physical examination revealed an irregular 3-cm ulcerative mass arising from the left tonsil (Fig 1). The rest of his oral cavity was otherwise normal and there was no cervical lymphadenopathy. Carcinoma of the left tonsil was suspected. Computed tomographic (CT) scan of the head and neck disclosed a non-specific soft tissue thickening and mucosal contrast enhancement at the left side of the oropharynx. Transoral punch biopsy of the left tonsillar mass was performed. Histopathology revealed no evidence of malignancy with heavy stromal infiltration by neutrophils, lymphocytes, and plasma cells plus focal microabscess formation. No granuloma or fungal elements were seen.

Subsequent upper gastrointestinal endoscopy was performed and disclosed the left tonsillar mass with no other abnormalities within the hypopharynx, oesophagus, or stomach. Because of the progressive odynophagia, a nasogastric feeding tube was inserted; as there was no improvement and the diagnosis was still elusive, left tonsillectomy was performed on the same day and was uneventful.

Histopathological examination of the left tonsil showed lymphoid tissue with preserved architecture present beneath the stratified squamous epithelium. Hyperplastic lymphoid follicles with germinal centre surrounded by mantle cells were present. Plasma cells were seen at the interfollicular area. There were no malignant cells. Immunostaining revealed more than $90 \%$ of plasma cells per high-power field with an immunoglobulin (Ig) G4:IgG ratio of more than $40 \%$ (Fig 2). The final diagnosis was IgG4-related disease of the left tonsil.

The patient refused workup with positron emission tomography-CT scan but clinically there was no feature of systemic IgG4-related disease so steroids were not prescribed. His odynophagia gradually improved and he could tolerate oral feeding with congee. The feeding nasogastric tube

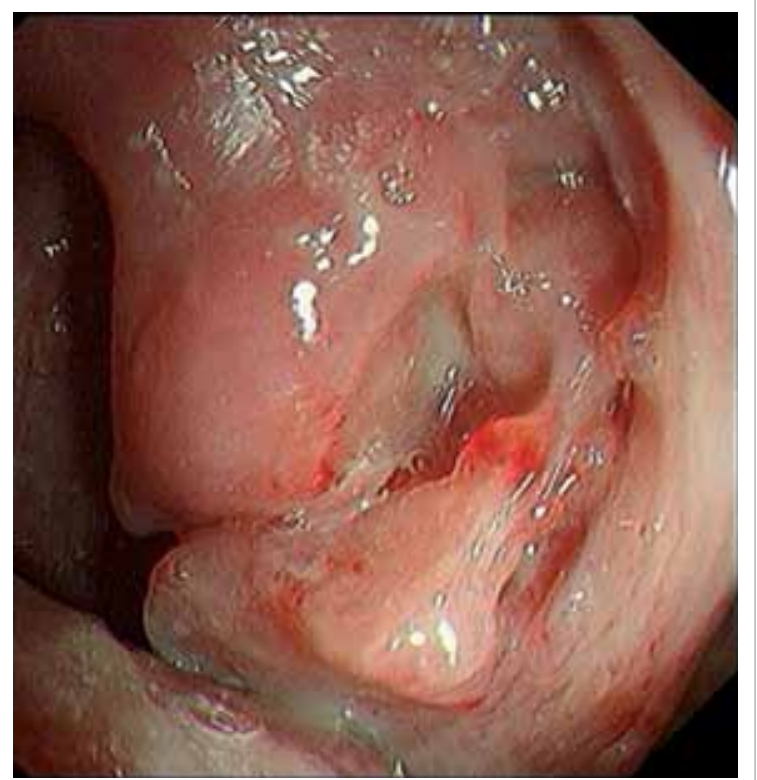

FIG I. Ulcerative left tonsillar mass with macroscopic features mimicking squamous cell carcinoma

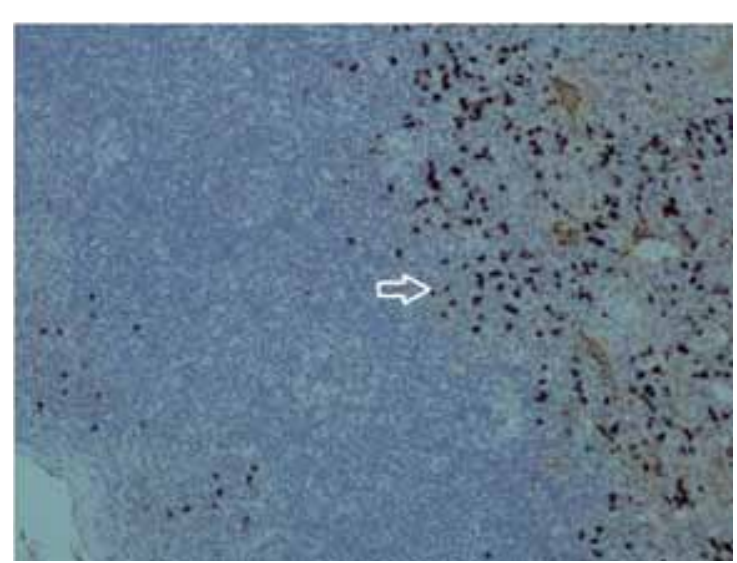

FIG 2. Immunohistochemical stain for immunoglobulin (Ig) G4 showing lgG4-positive plasma cells (arrow) [original magnification, $x$ 200]

was removed. Unfortunately, the patient had a depressive mood and general debility. He eventually died of pneumonia in September 2015. 


\section{Discussion}

Immunoglobulin G4-related disease is a fibroinflammatory condition often associated with elevated serum IgG4 level. ${ }^{1}$ It is a multi-organ entity that encompasses various conditions formerly considered to be unrelated, single-organ diseases. ${ }^{2}$ It is notorious for its resemblance to malignant disease such as carcinoma of the pancreas. It is a source of undue anxiety for both patients and clinicians as the diagnosis may be difficult if the index of clinical suspicion for IgG4-related disease is low. Systemic symptoms (asthenia, weight loss, or fever) may occur in a minority of patients. The disease can affect the pancreas (autoimmune pancreatitis with abdominal pain), biliary tree (sclerosing cholangitis with jaundice), salivary glands or lacrimal gland (parotid, submandibular and lacrimal gland enlargement), lymph nodes, kidneys (tubulointerstitial nephritis with renal failure), and retroperitoneum (ureteric stricture). ${ }^{2}$

Measuring the serum IgG4 level is useful to establish the diagnosis of this uncommon disease ${ }^{3}$ and may avoid unnecessary diagnostic surgery. Although serum IgG4 level was initially thought to be a key diagnostic feature of IgG4-related disease, more recent evidence has devalued the significance of a raised level. The key to diagnosis is immunohistochemical demonstration of tissue infiltration by IgG4-bearing plasma cells and morphological evidence of lymphoplasmacytic infiltrates, storiform fibrosis, and obliterative phlebitis. ${ }^{2}$ Serum IgG4 assay was not done as this investigation was not available at our hospital Nonetheless the diagnosis of IgG4-related disease of tonsil in this patient was obvious based on the histopathological examination with IgG4 immunostains.

Umehara et $\mathrm{al}^{4}$ proposed a set of criteria for the diagnosis of IgG4-related disease designed to be used irrespective of the specific organ involvement. The criteria are (1) serum IgG4 concentration of $>135 \mathrm{mg} / \mathrm{dL}$ and (2) >10 IgG-positive plasma cells demonstrated per high-power field, of which $>40 \%$ are IgG4-positive cells. Nevertheless the sensitivity is low for the diagnosis of autoimmune pancreatitis based on these criteria.

Of note, IgG4-related disease can manifest at the head and neck region. Kuttner's tumour of the submandibular gland has been well reported and often masquerades as carcinoma. ${ }^{5}$ Nonetheless IgG4-related disease within the oral cavity is very rare. To the best of our knowledge, only one case of simultaneous IgG4-related lesions affecting the left border of the tongue and right tonsil has been reported in the literature. ${ }^{6}$ This patient presented with an elevated and nodular mass devoid of surface ulceration, associated with a generalised skin rash. The oral and cutaneous lesions gradually resolved after therapy with steroid. ${ }^{6}$ In contrast, our patient had an ulcerative mass without any skin rash. Both cases were initially suspected to be squamous cell carcinoma because of the alarming clinical features.

Steroid remains the mainstay of treatment for IgG4-related disease. It should be started early in order to prevent tissue fibrosis and thus irreversible organ damage, except in cases of asymptomatic disease of the submandibular gland or lymph node. ${ }^{7}$ Radiological abnormalities often vanish following the commencement of steroid therapy. Unfortunately, disease relapse is not uncommon after steroid therapy is tapered. There is no evidence to support the effectiveness of adding conventional steroidsparing agents (eg azathioprine, methotrexate, or cyclophosphamide) to sustain remission for IgG4related disease. ${ }^{7}$ Although remission can be achieved in more than $80 \%$ of patients with induction steroid therapy, some clinicians support the use of maintenance steroid at $5 \mathrm{mg}$ or $2.5 \mathrm{mg}$ daily for a durable response. However, disease may flare in a quarter of patients despite maintenance therapy. Moreover, the optimal duration for maintenance treatment is uncertain and the morbidity associated with steroid, although in low dose, is not negligible. Fortunately, repeated induction therapy with steroid for disease relapse is normally effective. ${ }^{7}$

In summary, IgG4-related disease can involve the tonsils and masquerade as tonsil carcinoma. Clinicians should consider IgG4-related disease as one of the differential diagnoses for a suspicious tonsillar mass. Early diagnosis of tonsillar mass with biopsy is essential to exclude tonsil carcinoma and to allow prompt steroid treatment for IgG4-related disease of tonsil which is curable.

\section{References}

1. Deshpande V, Zen Y, Chan JK, et al. Consensus statement on the pathology of IgG4-related disease. Mod Pathol 2012;25:1181-92.

2. Stone JH, Brito-Zerón P, Bosch X, Ramos-Casals M. Diagnostic approach to the complexity of IgG4-related disease. Mayo Clin Proc 2015;90:927-39.

3. Hamano H, Kawa S, Horiuchi A, et al. High serum IgG4 concentrations in patients with sclerosing pancreatitis. $\mathrm{N}$ Engl J Med 2001;344:732-8.

4. Umehara H, Okazaki K, Masaki Y, et al. Comprehensive diagnostic criteria for IgG4-related disease (IgG4-RD), 2011. Mod Rheumatol 2012;22:21-30.

5. Chow TL, Chan TT, Choi CY, Lam SH. Kuttner's tumour (chronic sclerosing sialadenitis) of the submandibular gland: a clinical perspective. Hong Kong Med J 2008;14:46-9.

6. Khurram SA, Fernando M, Smith AT, Hunter KD. IgG4related sclerosing disease clinically mimicking oral squamous cell carcinoma. Oral Surg Oral Med Oral Pathol Oral Radiol 2013;115:e48-e51.

7. Khosroshahi A, Wallace ZS, Crowe JL, et al. International consensus guidance statement on the management and treatment of IgG4-related disease. Arthritis Rheumatol 2015;67:1688-99. 